J Hand Surg Am. 2017 August ; 42(8): 623-629.e1. doi:10.1016/j.jhsa.2017.05.006.

\title{
Diagnostic Testing Requested Before Surgical Evaluation for Carpal Tunnel Syndrome
}

\author{
Erika D. Sears, MD, MS ${ }^{1,2,3}$, Yu-Ting Lu, MPH ${ }^{1}$, Shannon M. Wood ${ }^{1}$, Jacob S. Nasser ${ }^{1}$, \\ Rodney A. Hayward, MD ${ }^{2,3,4}$, Kevin C. Chung, MD, MS ${ }^{1,3}$, and Eve A. Kerr, MD, MPH ${ }^{2,3,4}$ \\ ${ }^{1}$ Department of Surgery, Section of Plastic Surgery, University of Michigan Health System, Ann \\ Arbor, MI \\ ${ }^{2}$ Veterans Affairs Center for Clinical Management Research, VA Ann Arbor Healthcare System, \\ Ann Arbor, MI \\ ${ }^{3}$ Institute for Healthcare Policy and Innovation, University of Michigan, Ann Arbor, MI \\ ${ }^{4}$ Department of Internal Medicine, University of Michigan Health System, Ann Arbor, MI
}

\begin{abstract}
Purpose-We sought to evaluate how often physicians who perform carpal tunnel release (CTR) in the State of Michigan routinely request electrodiagnostic studies (EDS) or other diagnostic tests prior to an initial consultation, and whether provider or practice characteristics had an influence on requirements for pre-consultation diagnostic tests.
\end{abstract}

Methods-Through online data sources, we identified 356 providers in 261 practices throughout the State of Michigan with profiles confirming hand surgery practice or surgical treatment of carpal tunnel syndrome (CTS). We recorded American Society for Surgery of the Hand (ASSH) membership, teaching facility status, practice size, and primary specialty for each provider. Using a standardized telephone script, 219 providers were contacted by telephone to determine whether any diagnostic tests were needed before an appointment. Using multivariable logistic regression, we evaluated the relationship between the requirement for pre-consultation testing and surgeon and practice characteristics.

Results-Among the 134 providers who were confirmed to perform CTR, 57\% ( $\mathrm{n}=76)$ required and $9 \%(n=12)$ recommended a diagnostic test prior to the initial consultation. Of the 88 physicians who required/recommended testing, 85\% ( $\mathrm{n}=75)$ requested EDS, 22\% $(\mathrm{n}=19)$ requested an MRI, $13 \%(\mathrm{n}=11)$ requested a CT scan, and $9 \%(\mathrm{n}=8)$ requested an X-ray. Patients were asked to have multiple studies by 19 (22\%) of the 88 surgeons who requested/recommended testing. In

Corresponding Author: Erika Davis Sears, MD, MS, Veterans Affairs Center for Clinical Management Research, Section of Plastic Surgery, University of Michigan Health System, 2130 Taubman Center, SPC 5340, 1500 E. Medical Center Drive, Ann Arbor, MI 48109-5340, Phone: (734) 936-5895, Fax: (734) 763-5354, endavis@ med.umich.edu.

Publisher's Disclaimer: This is a PDF file of an unedited manuscript that has been accepted for publication. As a service to our customers we are providing this early version of the manuscript. The manuscript will undergo copyediting, typesetting, and review of the resulting proof before it is published in its final citable form. Please note that during the production process errors may be discovered which could affect the content, and all legal disclaimers that apply to the journal pertain.

Disclosure: None of the authors have any financial interest or conflicts of interest to declare in relation to the content of this article. 
the multivariable analysis, ASSH membership, size of practice and teaching facility status did not have a significant relationship with the requirement for pre-consultation testing.

Conclusions-Most surgeons who treat CTS in the State of Michigan routinely request EDS before evaluation, rather than reserving the test for cases where the diagnosis is unclear.

\section{Keywords}

Diagnostic tests; electrodiagnostic studies; carpal tunnel syndrome

\section{INTRODUCTION}

Electrodiagnostic Studies (EDS) are commonly used to evaluate patients with suspected carpal tunnel syndrome (CTS), with approximately $70 \%$ of American Society for Surgery of the Hand (ASSH) members reporting routine use of EDS prior to carpal tunnel release (CTR) in 2015. ${ }^{1}$ However, there is no reference standard diagnostic test for CTS. Current evidence consistently reports that information gathered from the history and physical exam has similar if not better sensitivity and specificity for CTS diagnosis compared to modalities such as EDS, MRI, and ultrasound, often with EDS having the highest false positive and false negative rates of the commonly used testing modalities. ${ }^{2-7}$ Although EDS is not associated with a high risk of harm, it is an unpleasant experience for patients. Furthermore, EDS may provide little value in patients who have classic symptoms and exam findings consistent with CTS, given the delay in surgery and added costs seen in patients who have EDS prior to CTR. ${ }^{8}$

The 2016 American Academy of Orthopaedic Surgeons (AAOS) Clinical Practice Guidelines (CPG) on the Management of Carpal Tunnel Syndrome supports the use of either EDS or a diagnostic questionnaire to aid the diagnosis of CTS. ${ }^{9}$ Although previous versions of the AAOS CPG recommended EDS for patients being considered for CTR, ${ }^{10}$ this recommendation does not appear in the latest guideline. The impact of previous recommendations on the routine use of EDS in current clinical practice for patients with suspected CTS is unknown. Furthermore, it is unknown how often surgeons are using EDS or other diagnostic tests prior to an initial patient evaluation, rather than ordering the tests selectively based on information obtained in the history and physical examination.

We sought to evaluate how often physicians who perform CTR in the State of Michigan routinely request EDS and/or other diagnostic tests prior to an initial consultation. Secondarily, we aimed to determine whether provider or practice characteristics had an influence on requirements for pre-consultation diagnostic tests. We hypothesized that EDS for CTS would be widely utilized before an initial consultation regardless of surgeon or practice factors.

\section{MATERIALS AND METHODS}

\section{Dataset and Study Selection}

This study received non-regulated status by the Institutional Review Board. We identified surgeons who potentially perform CTR in the State of Michigan through online data sources. 
ASSH members practicing in Michigan were identified using the ASSH membership directory. ${ }^{11}$ Non-ASSH members were identified by using two online physician search engines, DocSpot ${ }^{12}$ and HealthGrades. ${ }^{13}$ The search engines were chosen to compile a sample of physicians who potentially perform CTR by using related search terms unique to each site. The terms "hand surgery," "carpal tunnel release," and "carpal tunnel" were chosen to identify prospective providers on DocSpot and the terms "orthopaedic hand surgery," "plastic surgery of the hand," and "neuroplasty (limited to neurosurgeons)" were chosen to identify prospective providers on HealthGrades. We recorded publicly-available data from the physician profiles and verified each element on the physician's personal website when available, including the physician name, practice name, address, phone number for appointment scheduling, teaching facility status, size of the practice (the number of surgeons in the same practice performing CTR), and primary specialty (plastic surgery, orthopaedic surgery, neurosurgery, or general surgery). Providers were excluded if their profile or personal webpage did not indicate that they performed CTR or hand surgery. We identified 356 providers in 261 practices who met the inclusion criteria (Figure 1).

We categorized practices as solo $(\mathrm{N}=176)$, two-provider ( $\mathrm{N}=43$ practices), or group practices (3+ providers; $\mathrm{N}=42$ practices). We included all the identified group practices ( $\mathrm{N}=42$ practices) to allow evaluation of within-group practice variation. Furthermore, 58 solo or two-provider practices were randomly sampled to provide a total of 100 practices for inclusion (Figure 1). The sample size had sufficient power to detect differences in proportions of at least $25 \%$ between two groups for the outcome of recommending or requesting any diagnostic test, with power (beta) of 0.8 and alpha of 0.05 . Using a standardized telephone script (Appendix A), three trained research assistants contacted the office staff of providers who practiced in one of the 100 selected practices by telephone between June $28^{\text {th }}$ and July $15^{\text {th }}, 2016$. The assistants posed as a patient who was seeking to be seen for surgical evaluation for CTS and first verified that the physician/practice information was correct and that the provider performed CTR. The assistants asked whether any diagnostic tests were needed before an appointment with the provider and recorded the specific tests that were requested or recommended. Physicians who did not perform CTR $(\mathrm{N}=30)$ or who could not be reached by phone $(\mathrm{N}=55)$ were excluded from further analyses.

\section{Study Variables}

We extracted provider and practice characteristics from the online data sources and verified the characteristics during the phone calls to increase the completeness and accuracy of the recorded data. The provider characteristics recorded included ASSH membership and primary specialty, including plastic surgery, general surgery, orthopaedic surgery, and neurosurgery. The practice characteristics recorded included involvement in teaching and the number of providers in the practice. We categorized physicians working with residents or fellows as working in a teaching facility. The size of practice represented the number of surgeons in the same clinic who performed CTR. We recorded the reported requirements for pre-consultation diagnostic testing for each provider, including the need for EDS testing and/or alternative study (CT, MRI, ultrasound, X-ray). We categorized diagnostic testing requirements into the following three groups: test required, test recommended, and test not required. Diagnostic tests were defined as being required when the clinic requested that the 
caller have a test ordered by the surgeon or primary care provider prior to an initial evaluation. We defined the test as recommended if the clinic indicated that a test would be helpful prior to the initial appointment.

\section{Statistical Analysis}

We used descriptive statistics to summarize the prevalence of required pre-consultation tests. Among physicians performing CTR, we compared providers with no testing requirements to a combined group of providers who recommended or required pre-consultation studies. We used Fischer's exact test for unadjusted statistical comparisons of the association between patient and provider attributes with pre-consultation testing. Independent (adjusted) associations were estimated using multiple logistic regression. Adjusted predicted probabilities of pre-consultation testing (average marginal effects) were calculated using the multiple logistic model.

\section{RESULTS}

Of 477 physicians identified by the search strategy, 121 physicians who did not perform hand surgery or did not practice in Michigan were excluded. Among the 219 physicians from 100 solo or group practices contacted, the phone questionnaire could be completed for 207 (95\%) providers. Information could not be confirmed on the phone for 43 providers, 30 physicians did not perform CTR, and invalid phone numbers were present for 12 providers, all of whom were excluded from the final analysis. Among the 219 physicians contacted by phone, $61 \%(n=134)$ were confirmed to perform CTR (Figure 1).

Among the 134 providers who performed CTR, 57\% $(\mathrm{n}=76)$ required and $9 \%(\mathrm{n}=12)$ recommended a diagnostic test prior to the initial consultation. Only $34 \%(\mathrm{n}=46)$ of providers did not require or recommend any diagnostic testing prior to the initial consultation. Two clinics asked the caller about symptoms before requesting a diagnostic test. Respondent characteristics based on pre-consultation diagnostic testing requirements are outlined in Table 1. Among the 134 physicians who performed CTR, 55\% $(\mathrm{n}=28)$ of ASSH members and $72 \%(n=60)$ of non-ASSH members required or recommended diagnostic testing prior to the initial evaluation. In measuring practice differences by specialty, neurosurgeons were significantly more likely to require/recommend preconsultation tests (92\%) compared to orthopaedic and plastic surgeons (53\% and 59\%, respectively; $\mathrm{P}<0.05)$. No significant differences in pre-consultation testing recommendations were found by practice size or teaching status (Table 1). Among the nonsolo provider practices, 50\% (6/12 two-provider practices and 9/18 group practices) did not have any variation of diagnostic testing required between providers within the practice, meaning that all the surgeons in the practice had similar practices regarding pre-consultation testing recommendations.

The specific pre-consultation diagnostic tests required/recommended by surgeons are outlined in Table 2. Of the 88 physicians who required/recommended testing, $85 \%$ ( $\mathrm{n}=75$ ) requested EDS, 22\% $(\mathrm{n}=19)$ requested an MRI, 13\% $(\mathrm{n}=11)$ requested a CT scan, and 9\% $(n=8)$ requested an X-ray. Patients were asked to have multiple studies by $19(22 \%)$ of the 88 surgeons who requested/recommended testing. Furthermore, CT, MRI, or both were 
requested by nearly a quarter of providers who requested or recommended testing (Table 2). In the multivariable analysis, ASSH membership, size of practice and teaching facility status did not have a significant relationship with the requirement for pre-consultation testing. We found no significant difference in plastic surgeons and orthopaedic surgeons in preconsultation testing practices in the adjusted model. The neurosurgeon specialty was the only significant independent predictor associated with testing requirements (adjusted probability of $93 \%$ [95\% CI: $64 \%-99 \%$ ] of required/recommended pre-consultation testing, Table 3).

\section{DISCUSSION}

Although the 2016 AAOS CPG suggests that EDS and/or diagnostic questionnaires could be used to aid the diagnosis of CTS and no longer recommends routine use of EDS for patients undergoing CTR, the findings of this study suggest that the majority of surgeons who treat CTS in the State of Michigan request EDS before evaluation, rather than reserving the test for cases where the diagnosis is unclear. The requirement for pre-consultation testing was common regardless of ASSH member status, specialty, practice size, and teaching facility status. Furthermore, studies that are not recommended by the current and previous CPG for diagnosis of CTS (CT, MRI, or both) were requested or recommended by nearly a quarter of surgeons who requested tests.

Our findings support previous research suggesting that a substantial number of physicians routinely obtain EDS for patients undergoing CTR. ${ }^{1,14,15}$ Munns et al $^{1}$ found that $72 \%$ of hand surgeons routinely obtained preoperative EDS in a 2015 survey of 716 ASSH members. A 2014 survey of 705 ASSH members also found that 55\% of respondents believed EDS was usually necessary to provide sufficient justification to indicate a surgery in addition to a supporting history and physician exam. However, $64 \%$ of surgeons surveyed indicated that they were more likely to order EDS based on the CPG recommendations at the time, and most were concerned about medicolegal consequences created by the guidelines. ${ }^{14}$ Similarly, Shin et al ${ }^{15}$ found that $81 \%$ of respondents routinely ordered EDS prior to elective surgery for CTS in a 2012 survey of 123 members of the American Association for Hand Surgery. While these studies described high rates of EDS use for patients prior to surgery, no previous study examined whether providers ordered EDS prior to or after an initial consultation. The current study highlights the prevalent practice of indiscriminant EDS use among the surgeons in the study sample, rather than reserving the test to aid diagnosis for clinically uncertain cases.

Although the previous CPG suggested that EDS should be obtained if surgical management was being considered, ${ }^{10}$ we hypothesize that providers apply EDS more broadly to improve efficiency of establishing a treatment recommendation at the time of the initial evaluation. However, no test has been shown to be superior to clinical evaluation for diagnosis of CTS. For example, Graham ${ }^{6}$ found that EDS did not change the probability of CTS diagnosis for most patients who were diagnosed with CTS based on history and physician examination alone. Of 142 patients referred for upper extremity EDS evaluation, 73\% had a pretest probability of CTS diagnosis greater than 0.8 as estimated by the CTS- 6 diagnostic questionnaire. Among these patients with a high pretest probability of CTS diagnosis, the 
average change in probability of CTS diagnosis was 0.02 . In addition, EDS lowered the post-test probability of CTS diagnosis in patients who already had a low $(<0.5)$ pretest probability of diagnosis. In other words, EDS testing is unlikely to impact the probability of CTS diagnosis in patients with a high probability of diagnosis based on history and physical exam criteria.

A latent class analysis by Fowler ${ }^{2}$ showed that EDS had similar sensitivity (91\%) compared to ultrasound (US) and the CTS-6 diagnostic questionnaire (91\% and 95\%, respectively), whereas EDS had the lowest specificity (83\%) compared to US and CTS-6 (94\%, and 91\%, respectively). Similarly, a study comparing the diagnostic accuracy of EDS, US, CT and MRI in CTS, compared to clinical diagnosis as a reference standard, showed that these four tests had similar accuracy for the diagnosis of CTS. ${ }^{16}$ The existing evidence does not support using either EDS or imaging tests over history and physical exam for CTS diagnosis. Furthermore, EDS prior to CTR was associated with prolonged time to surgery, additional out of pocket costs, and greater total costs in a national study. ${ }^{8}$

This study had several limitations. The study sample included physicians limited to the State of Michigan and did not directly evaluate national practices. However, given that the overall rates of testing used prior to surgery was similar in our studies to other national surveys, we expect that our findings reflect national practices of using EDS and other diagnostic tests prior to CTS evaluation. The sample size was likely underpowered to detect small differences in provider and practice characteristics, but expanding the sample size would likely show a similarly common practice of requesting pre-consultation diagnostic tests. In addition, the study design prevented us from evaluating the impact of insurance type on the requirement for pre-consultation diagnostic tests. Furthermore, we do not know the reason that patients were asked to obtain diagnostic tests prior to a consultation and whether providers would offer surgery without prior testing. Some providers may advocate for routine EDS to measure severity of CTS or to predict improvement after surgical intervention, even if the test may not influence treatment recommendations. Others may routinely request diagnostic studies before evaluating a patient with CTS to establish a treatment plan at the time of the consultation and avoid the need for return visits with the surgeon. However, many patients likely receive diagnostic tests that have no bearing on the treatment plan. Although the study was conducted shortly after the new AAOS CPG were published, wide dissemination of new guidelines takes several months. It is possible that some surgeons in this study who requested pre-consultation EDS were influenced by the previous AAOS CPG that recommended EDS testing for patients being considered for surgery. The impact of diagnostic testing self-referral was outside the scope of this study, but also may influence provider practices given potential financial incentives. Lastly, it is possible that clerical staff could ask a patient to obtain a test even if the surgeon personally would not order a test. This may be more plausible in a group practice where individual surgeon practices differ. However, it is worth noting that the majority of office representatives ask patients to obtain tests before scheduling an appointment. It is unknown whether the support staff might be gatekeepers and inappropriately ask for tests in the interest of perceived office efficiency. Regardless of whether the office staff represent the true preferences of the surgeon, the requests that office staff make of patients calling for an 
appointment will have a direct impact on the tests that patients obtain before an appointment.

Despite these limitations, this study shows the prevalent practice of routinely requesting EDS and other diagnostic tests prior to CTS consultation. There is a need to highlight the change in the AAOS CPG and the responsibility of providers to advocate for more judicious EDS and diagnostic testing use. Given the cost and inconvenience of diagnostic tests, patients should be included in the decision to obtain tests if they are used for purposes other than informing treatment decisions. It is likely that a transition to bundled, episode-based payment for CTR will include any diagnostic testing performed. Greater clarity in the appropriateness of indications for EDS and other preoperative tests will help both primary care providers and surgeons to order tests that patients need, but not those from which patients are not likely to benefit. In the quest for high value care, providers must consider whether the benefit of the test likely outweighs the costs, inconvenience, and potential for treatment delay.

\section{Acknowledgments}

Support for this study was provided in part by the grants from the National Institute of Arthritis and Musculoskeletal and Skin Diseases of the National Institutes of Health under Award Number 2 K24-AR053120-06, a Midcareer Investigator Award in Patient-Oriented Research (to Dr. Kevin C. Chung). The content is solely the responsibility of the authors and does not necessarily represent the official views of the National Institutes of Health, the University of Michigan, or the Department of Veterans Affairs.

\section{References}

1. Munns JJ, Awan HM. Trends in carpal tunnel surgery: an online survey of members of the American Society for Surgery of the Hand. J Hand Surg Am. 2015; 40:767-771. e762. [PubMed: 25747738]

2. Fowler JR, Cipolli W, Hanson T. A Comparison of Three Diagnostic Tests for Carpal Tunnel Syndrome Using Latent Class Analysis. J Bone Joint Surg Am. 2015; 97:1958-1961. [PubMed: 26631997]

3. Grundberg AB. Carpal tunnel decompression in spite of normal electromyography. J Hand Surg Am. 1983; 8:348-349. [PubMed: 6875246]

4. Szabo RM, Slater RR Jr, Farver TB, Stanton DB, Sharman WK. The value of diagnostic testing in carpal tunnel syndrome. J Hand Surg Am. 1999; 24:704-714. [PubMed: 10447161]

5. Fowler JR, Munsch M, Tosti R, Hagberg WC, Imbriglia JE. Comparison of ultrasound and electrodiagnostic testing for diagnosis of carpal tunnel syndrome: study using a validated clinical tool as the reference standard. J Bone Joint Surg Am. 2014; 96:e148. [PubMed: 25187592]

6. Graham B. The value added by electrodiagnostic testing in the diagnosis of carpal tunnel syndrome. J Bone Joint Surg Am. 2008; 90:2587-2593. [PubMed: 19047703]

7. Werner RA, Andary M. Electrodiagnostic evaluation of carpal tunnel syndrome. Muscle Nerve. 2011; 44:597-607. [PubMed: 21922474]

8. Sears ED, Swiatek PR, Hou H, Chung KC. Utilization of Preoperative Electrodiagnostic Studies for Carpal Tunnel Syndrome: An Analysis of National Practice Patterns. J Hand Surg Am. 2016; 41:665-672. e661. [PubMed: 27068003]

9. American Academy of Orthopaedic Surgeons. [Accessed June 1, 2016] Management of Carpal Tunnel Syndrome Evidenced-Based Clinical Practice Guideline. 2016. www.aaos.org/ctsguideline

10. Keith MW, Masear V, Chung KC, et al. American Academy of Orthopaedic Surgeons Clinical Practice Guideline on diagnosis of carpal tunnel syndrome. J Bone Joint Surg Am. 2009; 91:24782479. [PubMed: 19797585]

11. [Accessed May 13, 2016] American Society for Surgery of the Hand (ASSH) Membership Directory. https://myaccount.assh.org/membershipdirectoryprofessional 
12. DOCSPOT. [Accessed June 1, 2016] https://www.docspot.com/

13. HealthGrades. [Accessed June 1, 2016] https://www.healthgrades.com/

14. Lane LB, Starecki M, Olson A, Kohn N. Carpal tunnel syndrome diagnosis and treatment: a survey of members of the American Society For Surgery of the Hand. J Hand Surg Am. 2014; 39:21812187. e2184. [PubMed: 25227597]

15. Shin EK, Bachoura A, Jacoby SM, Chen NC, Osterman AL. Treatment of carpal tunnel syndrome by members of the American Association for Hand Surgery. Hand (N Y). 2012; 7:351-356. [PubMed: 24294152]

16. Deniz FE, Oksuz E, Sarikaya B, et al. Comparison of the diagnostic utility of electromyography, ultrasonography, computed tomography, and magnetic resonance imaging in idiopathic carpal tunnel syndrome determined by clinical findings. Neurosurgery. 2012; 70:610-616. [PubMed: 21869718] 


\section{Appendix}

\begin{tabular}{l} 
Script for Carpal Tunnel Pre-Consultation Testing Requirement Inquiry \\
\hline Clinic/Hospital Information \\
$\mathrm{Hi}$, My name is XXX. I am calling to schedule an appointment with Dr. XXX. \\
[If clinic/hospital is CORRECT, GO TO Physician Information \#1] \\
[If INCORRECT, GO TO Alternate Clinic/Hospital Information]
\end{tabular}

Alternate Clinic/Hospital Information

Could you give me the phone number to schedule an appointment with Dr. XXX? Thank you.

[RECORD information and GO TO Request Alternate Physicians \#1]

Physician Information \#1

I am a new patient. I was diagnosed with carpal tunnel by my family doctor. Does Dr.XXX do carpal tunnel surgery?

[If answer is YES, GO TO Physician Information \#2]

[lf answer is NO, GO TO Request Alternate Physicians \#1]

\section{Physician Information \#2}

Dr. XXX is a plastic surgeon/general surgeon/orthopedic surgeon/neurosurgeon, correct?

[RECORD and VERIFY specialty, GO TO Confirm Facility Information]

\section{Request Alternate Physicians \#1}

Great thanks. Is there anyone else in the practice who does carpal tunnel surgery?

[RECORD the names]

Thank you. I will call back for an appointment another time. [End-call]

Confirm Facility Information

Just curious, Does Dr. XXX work with residents?

[RECORD answer (yes/no), GO TO Test Requirement]

\section{Test Requirement}

Do I need to have any tests done before the appointment?

[CHOOSE all that apply [EMG, CT, MRI, US, X-Ray], GO TO Request Appointment]

\section{Request Appointment}

Ok. It is always good to know. So, when is the earliest date to see Dr.XXX? (background info: I will be paying out of pocket because I have a very high deductible)

[RECORD appointment date and GO TO Request Alternate Physicians2]

\section{Request Alternate Physicians \#2}

Just curious, is there anyone else in the practice who does carpal tunnel surgery? Maybe I can see them sooner? Can you give me their names?

[RECORD names, if none leave it blank and GO TO The End]

\section{The End}

I will call you back to schedule I have to check my work schedule. Thank you very much for your help. [End-call]

Appendix A. 


\section{Clinical Relevance}

In the quest for high value care, providers must consider whether the benefit of diagnostic tests for carpal tunnel syndrome likely outweighs the costs, inconvenience, and potential for treatment delay. 


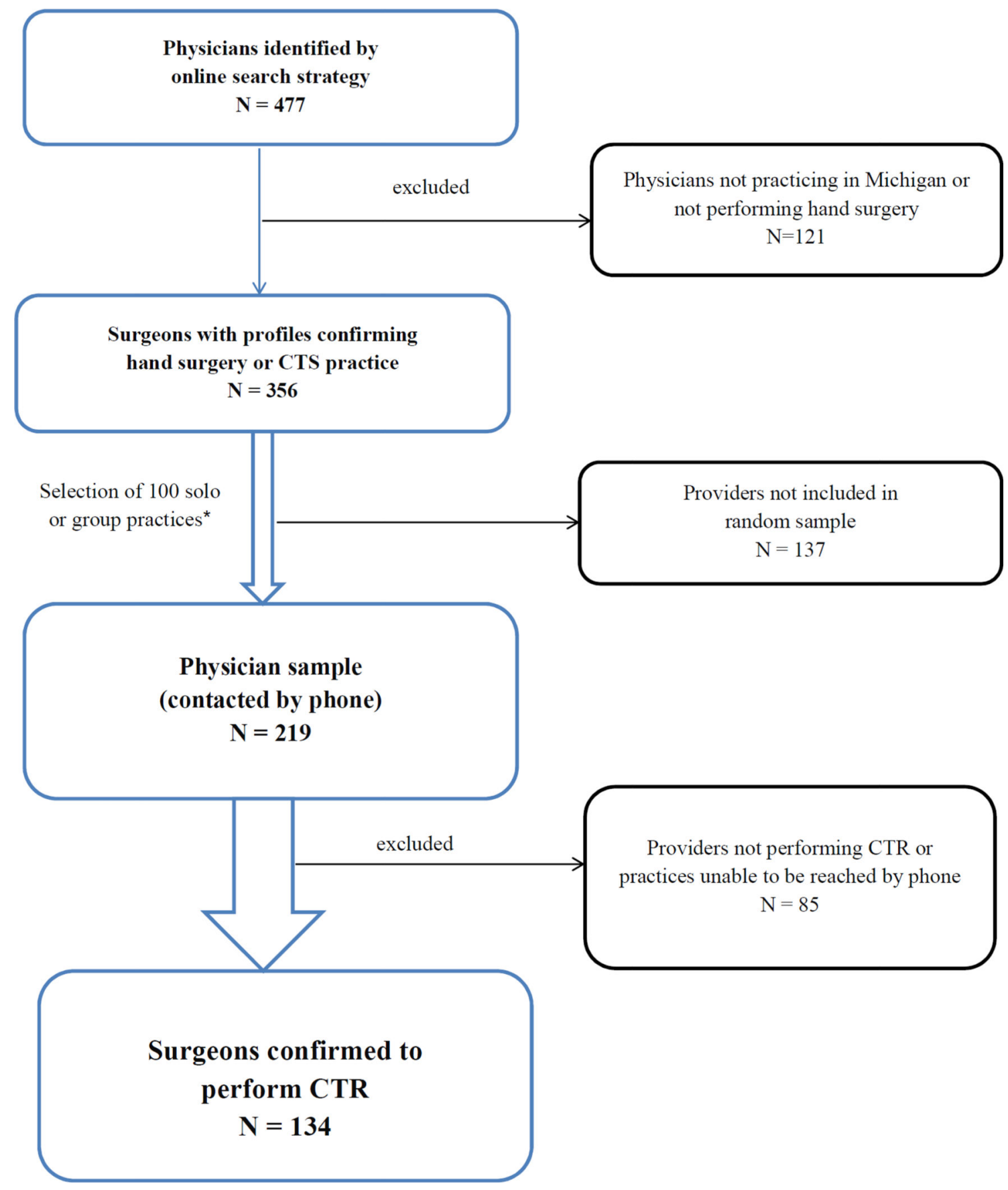

Figure 1.

Inclusion and Exclusion Criteria of Physician Sample Contacted by Phone. CTR: carpal tunnel release. $*$ All practices with 3 or more surgeons were included ( 150 surgeons from 42 practices). An additional 58 solo and two-provider practices were randomly sampled (69 surgeons from 58 solo or two-provider practices). 


\section{Table 1}

Provider Characteristics Based On Requirement for Diagnostic Testing Before Carpal Tunnel Syndrome Consultation

\begin{tabular}{|l|c|c|c|}
\hline & $\begin{array}{c}\text { Test Required/ } \\
\text { Recommended }\end{array}$ & Test Not Required & Total \\
\hline ASSH Member & $28(55 \%)$ & $23(45 \%)$ & 51 \\
Yes & $60(72 \%)$ & $23(28 \%)$ & 83 \\
No & & & \\
\hline Specialty ${ }^{*}$ & $2(100 \%)$ & $0(0 \%)$ & 2 \\
General Surgery & $33(92 \%)$ & $3(8 \%)$ & 36 \\
Neurosurgery & $34(53 \%)$ & $30(47 \%)$ & 64 \\
Orthopaedic Surgery & $19(59 \%)$ & $13(41 \%)$ & 32 \\
Plastic Surgery & & & \\
\hline Size of Practice & $25(65 \%)$ & $13(34 \%)$ & 38 \\
1 provider & $16(67 \%)$ & $8(33 \%)$ & 24 \\
2 providers & $47(65 \%)$ & $25(34 \%)$ & 72 \\
3+ providers & & & \\
\hline Teaching Facility & $47(65 \%)$ & $25(35 \%)$ & 72 \\
Yes & $41(66 \%)$ & $21(34 \%)$ & 62 \\
No & $\mathbf{8 8}$ & $\mathbf{4 6}$ & $\mathbf{1 3 4}$ \\
\hline Total & & & \\
\hline
\end{tabular}

P-value $<0.05$ in Fisher's Exact Test 
Table 2

Combinations of Pre-consultation Diagnostic Tests Required/Recommended for Carpal Tunnel Syndrome

\begin{tabular}{|l|c|c|c|c|}
\hline & ASSH Members & Non-ASSH Members & Total & Percent \\
\hline EDS only & 25 & 42 & 67 & $76 \%$ \\
MRI + CT & 0 & 6 & 6 & $7 \%$ \\
EDS + MRI & 1 & 2 & 3 & $3 \%$ \\
MRI + X-ray & 0 & 3 & 3 & $3 \%$ \\
EDS + MRI + CT & 1 & 1 & 2 & $2 \%$ \\
EDS + MRI + X-ray & 1 & 1 & 2 & $2 \%$ \\
MRI + CT + X-ray & 0 & 2 & 2 & $2 \%$ \\
MRI only & 0 & 1 & 1 & $1 \%$ \\
X-ray only & 0 & 1 & 1 & $1 \%$ \\
EDS + CT & 0 & 1 & 1 & $1 \%$ \\
\hline Total & $\mathbf{2 8}$ & $\mathbf{6 0}$ & $\mathbf{8 8}$ & $\mathbf{1 0 0 \%}$ \\
\hline
\end{tabular}

롤

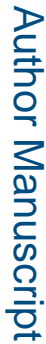

로을

J Hand Surg Am. Author manuscript; available in PMC 2018 August 01. 
Table 3

Multivariable Logistic Regression of Pre-Consultation Test Requirement ( $\mathrm{n}=132)^{*}$

\begin{tabular}{|c|c|c|c|}
\hline & Adjusted OR (95\% CI) & $\mathbf{P}$ & Predicted Probability \\
\hline \multicolumn{4}{|l|}{ ASSH Member } \\
\hline Yes & $0.82(0.29-2.28)$ & 0.71 & $0.70(0.45-0.87)$ \\
\hline No & Reference & & $0.74(0.56-0.86)$ \\
\hline \multicolumn{4}{|l|}{ Specialty } \\
\hline Neurosurgery & $14.08(2.86-69.38)$ & $<0.05$ & $0.93(0.64-0.99)$ \\
\hline Plastic Surgery & $1.37(0.40-4.76)$ & 0.64 & $0.56(0.21-0.86)$ \\
\hline Orthopaedic Surgery & Reference & & $0.48(0.26-0.72)$ \\
\hline \multicolumn{4}{|l|}{ Size of Practice } \\
\hline 1 provider & $0.34(0.09-1.34)$ & 0.13 & $0.60(0.35-0.81)$ \\
\hline 2 providers & $0.56(0.13-2.48)$ & 0.45 & $0.71(0.40-0.90)$ \\
\hline $3+$ providers & Reference & & $0.81(0.63-0.92)$ \\
\hline \multicolumn{4}{|l|}{ Teaching Facility } \\
\hline Yes & $0.89(0.29-2.77)$ & 0.84 & $0.70(0.50-0.85)$ \\
\hline No & Reference & & $0.73(0.51-0.87)$ \\
\hline
\end{tabular}

Multivariable logistic regression modeled outcome of requiring/recommending pre-consultation test prior to consultation. 